\title{
Analysis of the Saturated Electromagnetic Devices under DC Bias Condition by the Modified Harmonic Balance Finite Element Method
}

\author{
${ }^{1}$ Xiaojun Zhao, ${ }^{2}$ Junwei Lu, Senior Member IEEE, ${ }^{1}$ Lin Li, ${ }^{3}$ Zhiguang Cheng, ${ }^{1}$ Tiebing Lu \\ ${ }^{1}$ School of Electrical and Electronic Engineering, North China Electric Power University \\ Yonghua North Street, Baoding, 071000, China \\ 158748295@163.com \\ ${ }^{2}$ Griffith School of Engineering, Griffith University, Brisbane, 4111, Australia \\ j.lu@griffith.edu.au
}

\begin{abstract}
The modified harmonic balance finite element method was applied to investigate the DC biased problem in the saturated electromagnetic devices. The electromagnetic field was solved by the block Gauss-Seidel algorithm combined with the relaxation iterative method. The DC bias effects on magnetizing current and magnetic induction was analyzed by means of harmonic solution in multi-frequency domain. The computation is more efficient than the time-domain FEM and harmonic analysis can be achieved.
\end{abstract}

\section{INTRODUCTION}

The harmonic balance finite element method (HBFEM) proved to be suitable for solving the harmonic problem [1]. A related method via block decomposition of the system equation was also presented [2]. In the HVDC transmission system, direct current invades the transformer windings and threats the normal operation of electric network. Therefore DC bias effects on power transformers should be investigated by processing the DC component and considering coupled problems in calculation of the DC biased magnetic field.

\section{DECOMPOSED HARMONIC BALANCED SYSTEM EQUATION}

The vector potential equation can be used to describe the nonlinear magnetic field.

$$
\nabla \times(v \nabla \times A)=J
$$

Since electromagnetic devices are excited simultaneously by direct and alternating current, the steady state variables $\boldsymbol{x}$ are expressed by a triangular series,

$$
x(t)=x_{0}+\sum_{n=1}^{\infty}\left(x_{2 n-1} \sin n \omega t+x_{2 n} \cos n \omega t\right)
$$

The harmonic finite element system matrix of HBFEM can be obtained by substituting (2) into (1),

$$
D_{i, i} A_{i}+N_{i} A_{i}=\sum_{j \neq i}^{\infty}-D_{i, j} A_{j}+G_{i} \quad(i, j=1,2,3 \ldots)
$$

$D_{i, i}$ and $N_{i}$ represent self reluctivity matrix and harmonic matrix respectively for the $i$-th harmonic solution of magnetic vector potential $A_{i}$. $D_{i, j}$ means the mutual reluctivity related to the $i$-th and $j$-th harmonic. $G_{i}$ is obtained from the spatial distribution of $i$-th harmonic component of current density.

As electromagnetic devices are excited by voltages, the electric potential difference in the coil region can be treated as line integral of electric potential gradient,

$$
V_{k, i}=\int_{l_{k}}(-\nabla \varphi) d l=R_{k} J_{k, i} S_{k}+\int_{l_{k}}\left(\partial A_{i} / \partial t\right) d l
$$

The subscripts $k$ and $i$ indicate circuit number and harmonic number respectively. Combined with finite element method, the corresponding matrix representation can be given,

$$
V_{k, i}=M_{k} J_{k, i}+C_{k, i} A_{i}
$$

\section{MODIFIED HARMONIC BALANCE FINITE ELEMENT METHOD}

The harmonic solutions of magnetizing currents and magnetic vector potentials are obtained by solving (3) and (5),

$$
\begin{gathered}
{\left[\begin{array}{cc}
Q_{i} & G_{k, i} \\
C_{k, i} & M_{k}
\end{array}\right]\left\{\begin{array}{c}
A_{i} \\
J_{k, i}
\end{array}\right\}=\left\{\begin{array}{c}
F_{i} \\
V_{k, i}
\end{array}\right\}} \\
F_{i}=\sum_{j \neq i}^{\infty}-D_{i, j} A_{j}
\end{gathered}
$$

$Q_{i}$ is the sum of matrices $D_{i, i}$ and $N_{i}$. The term $D_{i, i}$ is diagonally dominant in system matrix in the traditional HBFEM and relatively weak relations exist among all harmonic components of solutions. Therefore, the block Gauss-Seidel algorithm can be used to solve the separated harmonic balance finite element equations. Compared with the traditional HBFEM, the realistic computation by the modified method benefits from the reduced memory requirement, meanwhile the computational time is unaffected.

The modified method is applied to investigate the mechanism of DC biased phenomenon in an Epstein framelike core model and a three-phase power transformer. Magnetizing currents shown in Fig.1 are calculated under different DC bias. The fundamental frequency is $50 \mathrm{~Hz}$.

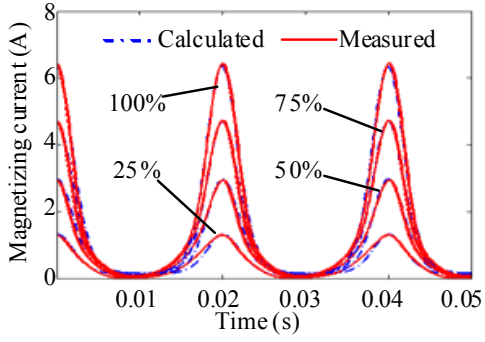

Fig. 1. Magnetizing currents under different DC bias

\section{REFERENCES}

[1] S. Yamada, K. Bessho, "Harmonic field calculation by the combination of finite element analysis and harmonic balance method," IEEE Transactions on Magnetics, Vol. 24, No. 6, pp. 2588-2590, November 1988.

[2] S. Yamada, P. P. Biringer, K. Bessho, "Calculation of nonlinear eddy current problems by the harmonic balance finite element method," IEEE Transactions on Magnetics, Vol. 27, No. 5, pp. 4122-4125, September 1991. 